[Original]

\title{
Effect of Jute Dust Exposure on Ventilatory Function and the Pertinence of Cough and Smoking to the Response
}

\author{
B. P. Ghattopadhyay, Sk. Jane Alam, P. K. Gangopadhyay and H. N. Saiyed \\ Regional Occupational Health Centre (Eastern). Calcutta-700 016, India
}

Abstract: The ventilatory capacity of 32 men exposed to jute dust in the jute industry, was estimated at the beginning and end of shifts on the first day and last day of a working week. A detailed occupational, clinical and smoking history was recorded and a more detailed questionnaire on respiratory symptoms was completed prior to the pulmonary function tests. The presence of a productive cough among workers was noted. A mean decrease of forced expiratory volume at one second $\left(\mathrm{FEV}_{1}\right)$ was observed among workers according to processes, concentration of dust exposure and smoking habit on the first day and last day of the week. The mean difference of values was observed in most of the comparisons but only a few were statistically significant. Low mean values of $\mathrm{FEV}_{1}$ were observed in workers having high dust exposure, a smoking history and productive cough in comparison to the low dust exposure group, non-smokers and workers without cough symptoms. The significant deterioration of $\mathrm{FEV}_{1}$ was found between before shift and after shift values of the low dust exposed group of workers who had a cough and smoking history. The relevence of these factors on the ventilatory function is observed and discussed.

Key words: jute dust, occupational exposure, shift work, pulmonary function test.

(Receired 31 October 1994, accepted 24 February 1995)

\section{Introduction}

In Eastern India, the jute industry plays a very important role among other industries. A major fraction of the population is involved in it. Like the cotton industry one other textile industry producing dust is the manufacturer of jute. Up till now very little has been known about the respiratory effects of jute dust, although a brief reference has been made to it by Arlidge and Schilling [1,2].

Jute fibre is obtained from the bark of the two cultivated species of the Tiliaceae, that is, the genus corchorus capsularis and corchorus olitorius. It is usually grown in soils of alluvial origin. The most improtant use of jute is 
for making fabrics for containers in which a large variety of commodities are transported or stored. It is also used in the making of carpets.

Jute dust is a non-toxic dust [3]. Most particles are large enough to be trapped in the nose and upper respiratory system. Only the small dust particles that reach the lungs contribute to the causes of certain non-specific lung diseases. Workers in the jute industry (as in other textile trades) are liable to contact "mill fever" especially at the beginning of employment. The condition lasts two to three days and frequently clears up without treatment. Byssinosis has not been identified in jute workers [3], even classical symptoms of byssinosis did not occur in those who were exposed to jute dust [4]. Siddhu et al. [3] and Mair et al., [5] found no jute effects which caused byssinosis. Only Popa and co-workers [6] suggested that byssionsis could result from exposure to jute dust. Gandevia and Milne [7] stated that the ventilatory capacity of 46 workers exposed to jute dust showed a significant decrease in the $\mathrm{FEV}_{1}$ on the first day of work. A subsequent study carried out by Valic and Zuskin [8], compared the effects of cotton and jute dust on respiratory symptoms in 60 cotton and 91 jute, non-smoking female workers of similar age and similar length of dust exposure. They showed that cotton workers had a significantly higher prevalence of byssinosis and of dyspnoea than did jute workers. But both the cotton and jute caused a significant reduction in ventilatory capacity on the first working shift of the week, with cotton dust having a significantly greater effect. Thus it showed that jute probably does lead to a shift decrement in the $\mathrm{FEV}_{1}$ but its effect is much less than that of cotton and flax and may be non-specific. Morgan and Seaton [9] have reported similar chest symptoms like byssinosis subjects in jute and sisal workers but there is a controversy whether these textiles cause byssinosis or not.

The present study was undertaken with a view to establish the ventilatory capacity of 32 workers exposed to jute dust at the beginning and at the end of the shift on the first and last day of a working week. Smoking habit, duration of exposure in the jute mill and presence of a productive cough and productive phlegm was also considered in each case to interpret their values in these aspects.

\section{Materials and Methods}

Our observation was made on male jute mill workers. The volunteers for the present study were taken from two jute mills and were between the age, height, weight and body surface area range of $20-58 \mathrm{yr}, 157-167 \mathrm{~cm}, 49-$ $56 \mathrm{~kg}, 1.49-1.60 \mathrm{~m}^{2}$ respectively. These subjects belonged to Batching, Spinning and Weaving departments. They were not suffering from any 
respiratory diseases. The mean age duration of exposure of the batching, spinning and weaving workers were $43.08 \pm 6.28,39.20 \pm 12.00,42.20 \pm 10.87$ years and $21.16 \pm 5.04,19.93 \pm 4.54,20.40 \pm 5.85$ years respectively. The ventilatory function was done by a dry wedge bellows spirometer, and the vitalograph and peak expiratory flow rate by Wright's peak flow meter. The same instrument and technical hand recorded PFT throughout the study. All subjects were well motivated before the final recordings were taken. Pulmonary function measurements were made in a comfortable standing posture with a nose clip. The functional parameters used in this study were forced vital capacity $(\mathrm{FVC})$, forced expiratory volume in one second $\left(\mathrm{FEV}_{1}\right)$, forced expiratory flow rate at $27-75 \%$ of $\mathrm{FVC}$ and peák expiratory flow rate (PEFR). The recordings were taken twice a day after a weekly break on Monday and the following Saturday. The first recording was taken just before starting their work as a before shift value and the second recording was taken at the end of their shift on the same day as an after shift value. Similar recordings were also taken on the last day of the week that is on Saturday. The symbols A, B, C and D were allotted to the values for Monday morning, Monday afternoon, Saturday morning and Saturday afternoon respectively. The work processes and each man's job were reviewed as Batching (Selection, Softening, Carding and Preparing), Spinning and Weaving. Batching is considered to be the most dusty kind of work followed by spinning and weaving.

The concentration of the jute dust in the work environment of the above said two jute mills was measured [10]. The prescribed threshold limit value (TLV) for nuisance dust was $10 \mathrm{mg} / \mathrm{m}^{3}$ stated by ACGIH, 1986-87 [11]. The highest concentration of the dust was found in the softening (batching) department (Mill I-13.39 \pm 3.92 , Mill II-10.85 \pm 5.60 ) which was above that level. The batching process as a whole was considered as a high dust zone $\left(8.87 \mathrm{mg} / \mathrm{m}^{3}\right.$ and $\left.6.86 \mathrm{mg} / \mathrm{m}^{3}\right)$ of the two jute mills. Workers studied from this process were considered as a high dust exposed group of workers and the workers of spinning and weaving were considered as in a low dust zone (1.50 $\mathrm{mg} / \mathrm{m}^{3}$ and $\left.1.06 \mathrm{mg} / \mathrm{m}^{3}\right)$. These workers were considered as the low dust exposed group of workers. In the high dust zone the dust contained more mineral matters and coarse vegetable fibres of raw jute. The abrupt fall of the dust level in these mills to beyond the preparing stage has been termed as "low dust zone". It is accounted that the fibre fraction of jute dust is more and mineral matter is less in this region [10].

Among these workers 15 subjects were non-smokers and 17 were smokers. Smoker subjects had a smoking history of 15 to $20 \mathrm{bidi} / \mathrm{cig}$. (or combined) per day. Details of the occupational and smoking histories of the subjects 
were recorded by following a standard questionnaire. Lung volumes obtained were expressed in body temperature on the atmospheric pressure of air saturated with water vapour (BTPS). Body height and body weight were measured in barefeet on a standard scale. The body surface area was calculated by using the Du-Bois and Du-Bois formula [12] .

\section{Results}

Pulmonary function test (PET) results on the first working day of the week after a weekend rest at the before shift and after shift period are presented in Table 1, similarly PFT results of the last day of the same week are depicted in Table 2. In both tables a comparison has been made between the before shift and after shift values of all PFT parameters. In each comparison, little mean difference of values was found which are statistically non-significant. Tables 3 to 9 set out the results of statistical analyses based on the change in $\mathrm{FEV}_{1}$. In the text, reference to the relevant statistical data is made by means of the number in the left hand column of the appropriate table and term "Significant" implies ' $P$ ' in the right hand column. In Table 3, the

Table 1. Mean values for $\mathrm{FVC}_{1} \mathrm{FEV}_{1}, \mathrm{FEF}_{25-75 \%}$ and PEFR of the first day of work

\begin{tabular}{|c|c|c|c|c|c|c|c|c|}
\hline & \multicolumn{4}{|c|}{ First day before shift } & \multicolumn{4}{|c|}{ First day after shift } \\
\hline & $\begin{array}{l}\text { FVC } \\
(\ell)\end{array}$ & $\begin{array}{c}\mathrm{FEV}_{1} \\
(\ell)\end{array}$ & $\begin{array}{c}\mathrm{FEF}_{25-75 \%} \\
(\ell / \mathrm{sec})\end{array}$ & $\begin{array}{l}\text { PEFR } \\
(\ell / \min )\end{array}$ & $\begin{array}{l}\text { FVG } \\
(\ell)\end{array}$ & $\begin{array}{c}\mathrm{FEV}_{1} \\
(\ell)\end{array}$ & $\begin{array}{c}\mathrm{FEF}_{25-75 \%} \\
(\ell / \mathrm{sec})\end{array}$ & $\begin{array}{c}\text { PEFR } \\
(\ell / \min )\end{array}$ \\
\hline $\begin{array}{l}\text { Batching } \\
\mathrm{n}=12\end{array}$ & $\begin{array}{r}2.77 \\
\pm 0.87\end{array}$ & $\begin{array}{r}1.93 \\
\pm 0.83\end{array}$ & $\begin{array}{r}1.37 \\
\pm 0.73\end{array}$ & $\begin{array}{c}381 \\
\pm 96.62\end{array}$ & $\begin{array}{r}2.77 \\
\pm 0.62\end{array}$ & $\begin{array}{r}1.94 \\
\pm 0.66\end{array}$ & $\begin{array}{r}1.43 \\
\pm 0.81\end{array}$ & $\begin{array}{c}380 \\
\pm 95.47\end{array}$ \\
\hline $\begin{array}{l}\text { Spinning } \\
\mathrm{n}=5\end{array}$ & $\begin{array}{r}3.05 \\
\pm 0.81\end{array}$ & $\begin{array}{r}2.37 \\
\pm 0.56\end{array}$ & $\begin{array}{r}1.88 \\
\pm 0.42\end{array}$ & $\begin{array}{c}475 \\
\pm 49.11\end{array}$ & $\begin{array}{r}2.93 \\
\pm 0.91\end{array}$ & $\begin{array}{r}2.27 \\
\pm 0.62\end{array}$ & $\begin{array}{r}1.89 \\
\pm 0.57\end{array}$ & $\begin{array}{c}468 \\
\pm 50.57\end{array}$ \\
\hline $\begin{array}{l}\text { Weaving } \\
\mathrm{n}=15\end{array}$ & $\begin{array}{r}3.41 \\
\pm 0.45\end{array}$ & $\begin{array}{r}2.64 \\
\pm 0.48\end{array}$ & $\begin{array}{r}2.44 \\
\pm 0.98\end{array}$ & $\begin{array}{c}481 \\
\pm 91.54\end{array}$ & $\begin{array}{r}3.16 \\
\pm 0.54\end{array}$ & $\begin{array}{r}2.52 \\
\pm 0.56\end{array}$ & $\begin{array}{r}2.39 \\
\pm 0.94\end{array}$ & $\begin{aligned} & 478 \\
\pm & 101.92\end{aligned}$ \\
\hline
\end{tabular}

Table 2. Mean values for $\mathrm{FVC}, \mathrm{FEV}_{1}, \mathrm{FEF}_{25-75 \%}$ and PEFR of the last day of work

\begin{tabular}{|c|c|c|c|c|c|c|c|c|}
\hline & \multicolumn{4}{|c|}{ Last day before shift } & \multicolumn{4}{|c|}{ Last day after shift } \\
\hline & $\begin{array}{l}\text { FVG } \\
(\ell)\end{array}$ & $\begin{array}{c}\mathrm{FEV}_{1} \\
(\ell)\end{array}$ & $\begin{array}{c}\mathrm{FEF}_{25-75 \%} \\
(\ell / \mathrm{sec})\end{array}$ & $\begin{array}{c}\text { PEFR } \\
(\ell / \min )\end{array}$ & $\begin{array}{l}\text { FVC } \\
(\ell)\end{array}$ & $\begin{array}{c}\mathrm{FEV}_{1} \\
(\ell)\end{array}$ & $\begin{array}{c}\mathrm{FEF}_{25-75 \%} \\
(\ell / \mathrm{sec})\end{array}$ & $\begin{array}{c}\text { PEFR } \\
(\ell / \min )\end{array}$ \\
\hline $\begin{array}{l}\text { Batching } \\
n=12\end{array}$ & $\begin{array}{r}2.76 \\
\pm 0.69\end{array}$ & $\begin{array}{r}1.83 \\
\pm 0.76\end{array}$ & $\begin{array}{r}1.17 \\
\pm 0.62\end{array}$ & $\begin{aligned} & 362 \\
\pm & 100.73\end{aligned}$ & $\begin{array}{r}2.58 \\
\pm 0.72\end{array}$ & $\begin{array}{r}1.76 \\
\pm 0.77\end{array}$ & $\begin{array}{r}1.22 \\
\pm 0.78\end{array}$ & $\begin{aligned} & 358 \\
+ & 114.10\end{aligned}$ \\
\hline $\begin{array}{l}\text { Spinning } \\
\mathrm{n}=5\end{array}$ & $\begin{array}{r}2.90 \\
\pm 0.87\end{array}$ & $\begin{array}{r}2.19 \\
\pm 0.59\end{array}$ & $\begin{array}{r}2.31 \\
\pm 1.58\end{array}$ & $\begin{array}{c}494 \\
\pm 53.78\end{array}$ & $\begin{array}{r}2.86 \\
\pm 0.80\end{array}$ & $\begin{array}{r}2.21 \\
\pm 0.59\end{array}$ & $\begin{array}{r}1.92 \\
\pm 0.61\end{array}$ & $\begin{array}{c}493 \\
\pm 60.80\end{array}$ \\
\hline $\begin{array}{l}\text { Weaving } \\
\mathrm{n}=15\end{array}$ & $\begin{array}{r}3.22 \\
\pm 0.54\end{array}$ & $\begin{array}{r}2.44 \\
\pm 0.55\end{array}$ & $\begin{array}{r}1.96 \\
\pm 0.86\end{array}$ & $\begin{array}{c}478 \\
\pm 98.53\end{array}$ & $\begin{array}{r}3.18 \\
\pm 0.63\end{array}$ & $\begin{array}{r}2.48 \\
\pm 0.60\end{array}$ & $\begin{array}{r}2.31 \\
\pm 1.09\end{array}$ & $\begin{array}{c}480 \\
\pm 98.85\end{array}$ \\
\hline
\end{tabular}


Table 3. Changes in $\mathrm{FEV}_{1}$ for the entire series according to different departments

\begin{tabular}{|c|c|c|c|c|c|c|}
\hline $\begin{array}{l}\text { Name of } \\
\text { Process }\end{array}$ & Decrease in $\mathrm{FEV}_{1}$ & $\overline{\mathrm{X}}$ & SD & $\mathrm{n}$ & $t$ & $P$ \\
\hline \multirow{4}{*}{$\begin{array}{l}\text { Batching } \\
n=12\end{array}$} & 1. $\mathrm{A} \rightarrow \mathrm{B}$ & -0.116 & 0.379 & 12 & 1.058 & $<0.40>0.30$ \\
\hline & 2. $\mathrm{C} \rightarrow \mathrm{D}$ & 0.069 & 0.175 & 12 & 1.368 & $<0.20>0.10$ \\
\hline & 3. $\mathrm{A} \rightarrow \mathrm{D}$ & 0.166 & 0.489 & 12 & 1.175 & $<0.30>0.20$ \\
\hline & 4. $\mathrm{A} \rightarrow \mathrm{C}$ & 0.096 & 0.513 & 12 & 0.652 & $<0.60>0.50$ \\
\hline \multirow{4}{*}{$\begin{array}{l}\text { Spinning } \\
\mathrm{n}=5\end{array}$} & 1. $\mathrm{A} \rightarrow \mathrm{B}$ & 0.096 & 0.149 & 5 & 1.435 & $<0.30>0.20$ \\
\hline & 2. $\mathrm{C} \rightarrow \mathrm{D}$ & 0.012 & 0.052 & 5 & 0.515 & $<0.70>0.60$ \\
\hline & 3. $\mathrm{A} \rightarrow \mathrm{D}$ & 0.158 & 0.112 & 5 & 3.147 & $<0.05>0.02$ \\
\hline & 4. $\mathrm{A} \rightarrow \mathrm{C}$ & 0.176 & 0.073 & 5 & 5.399 & $<0.01>0.001$ \\
\hline \multirow{4}{*}{$\begin{array}{l}\text { Weaving } \\
\mathrm{n}=15\end{array}$} & 1. $\mathrm{A} \rightarrow \mathrm{B}$ & 0.113 & 0.245 & 15 & 1.792 & $<0.10>0.05$ \\
\hline & 2. $\mathrm{C} \rightarrow \mathrm{D}$ & -0.037 & 0.221 & 15 & 0.653 & $<0.60>0.50$ \\
\hline & 3. $\mathrm{A} \rightarrow \mathrm{D}$ & 0.165 & 0.185 & 15 & 2.962 & $<0.02>0.01$ \\
\hline & 4. $\mathrm{A} \rightarrow \mathrm{C}$ & 0.185 & 0.212 & 15 & 3.388 & $<0.01>0.001$ \\
\hline
\end{tabular}

$\mathrm{A}$ and $\mathrm{B} \rightarrow$ First day (Monday) before shift and First day after shift.

$\mathrm{C}$ and $\mathrm{D} \rightarrow$ Last day (Saturday) before shift and Last day after shift.

$\overline{\mathrm{X}}=$ Mean value, $\quad \mathrm{SD}=$ Standard deviation, $\quad \mathrm{n}=$ Number of workers, $t={ }^{\prime} t$ 'value, $\quad P=$ Level of significance.

Table 4. Changes in $\mathrm{FEV}_{1}$ in relation to dust exposure

\begin{tabular}{|c|c|c|c|c|c|c|}
\hline $\begin{array}{l}\text { Sr. } \\
\text { No. }\end{array}$ & Decrease in $\mathrm{FEV}_{1}$ & $\overline{\mathrm{X}}$ & $\mathrm{SD}$ & $\mathrm{n}$ & $t$ & $P$ \\
\hline 1. & $\begin{array}{l}\mathrm{A} \rightarrow \mathrm{B} \\
\text { Low dust exposure }\end{array}$ & 0.109 & 0.221 & 20 & 2.199 & $<0.05>0.02$ \\
\hline 2. & $\begin{array}{l}\mathrm{A} \rightarrow \mathrm{B} \\
\text { High dust exposure }\end{array}$ & -0.116 & 0.380 & 12 & 1.058 & $<0.40>0.30$ \\
\hline 3. & $\begin{array}{l}\mathrm{C} \rightarrow \mathrm{D} \\
\text { Low dust exposure }\end{array}$ & -0.025 & 0.188 & 20 & 0.595 & $<0.60>0.50$ \\
\hline 4. & $\begin{array}{l}\mathrm{C} \rightarrow \mathrm{D} \\
\text { High dust exposure }\end{array}$ & 0.069 & 0.175 & 12 & 1.368 & $<0.20>0.10$ \\
\hline 5. & $\begin{array}{l}\mathrm{A} \rightarrow \mathrm{D} \\
\text { Low dust exposure }\end{array}$ & 0.163 & 0.193 & 20 & 3.793 & $<0.01>0.001$ \\
\hline 6. & $\begin{array}{l}\mathrm{A} \rightarrow \mathrm{D} \\
\text { High dust exposure }\end{array}$ & 0.166 & 0.489 & 12 & 1.175 & $<0.30>0.20$ \\
\hline 7. & $\begin{array}{l}\mathrm{A} \rightarrow \mathrm{C} \\
\text { Low dust exposure }\end{array}$ & 0.183 & 0.253 & 20 & 3.297 & $<0.01>0.001$ \\
\hline 8. & $\begin{array}{l}\mathrm{A} \rightarrow \mathrm{C} \\
\text { High dust exposure }\end{array}$ & 0.097 & 0.513 & 12 & 0.652 & $<0.60>0.50$ \\
\hline
\end{tabular}

$\mathrm{A}$ and $\mathrm{B} \rightarrow$ First day (Monday) before shift and First day after shift.

$\mathrm{C}$ and $\mathrm{D} \rightarrow$ Last day (Saturday) before shift and Last day after shift.

$\overline{\mathrm{X}}=$ Mean value, $\quad \mathrm{SD}=$ Standard deviation, $\quad \mathrm{n}=$ Number of workers, $t=$ ' $t$ 'value, $\quad P=$ Level of significance.

results of $\mathrm{FEV}_{1}$ of workers according to different processes are presented. In the batching department no significant difference was noticed when the values of before shift and after shift were compared, but in the weaving and spinning departments significant changes were found when the values of Monday before shift and Saturday after shift and Monday before shift and Saturday before shift 
were compared.

Table 4 represents the changes of $\mathrm{FEV}_{1}$ due to dust exposure. In the present study 20 workers belonged to the low dust exposure group and 12

Table 5. Changes in $\mathrm{FEV}_{1}$ in relation to dust exposure

\begin{tabular}{|c|c|c|c|c|c|c|}
\hline $\begin{array}{l}\text { Sr. } \\
\text { No. }\end{array}$ & Decrease in $\mathrm{FEV}_{1}$ & $\tilde{\mathrm{X}}$ & SD & $\mathrm{n}$ & $t$ & $P$ \\
\hline 1. & $\begin{array}{l}\mathrm{A} \rightarrow \mathrm{B} \\
\text { Low dust exposure with cough }\end{array}$ & 0.021 & 0.054 & 7 & 1.038 & $<0.40>0.30$ \\
\hline 2. & $\begin{array}{l}\mathrm{A} \rightarrow \mathrm{B} \\
\text { High dust exposure with cough }\end{array}$ & 0 & 0.351 & 6 & 0 & NS \\
\hline 3. & $\begin{array}{l}\mathrm{C} \rightarrow \mathrm{D} \\
\text { Low dust exposure with cough }\end{array}$ & 0.037 & 0.116 & 7 & 0.843 & $<0.50>0.40$ \\
\hline 4. & $\begin{array}{l}\mathrm{C} \rightarrow \mathrm{D} \\
\text { High dust exposure with cough }\end{array}$ & 0.043 & 0.230 & 6 & 0.461 & $<0.70>0.60$ \\
\hline 5. & $\begin{array}{l}\mathrm{A} \rightarrow \mathrm{D} \\
\text { Low dust exposure with cough }\end{array}$ & 0.145 & 0.095 & 7 & 4.06 & $<0.01>0.001$ \\
\hline 6. & $\begin{array}{l}\mathrm{A} \rightarrow \mathrm{D} \\
\text { High dust exposure with cough }\end{array}$ & 0.221 & 0.427 & 6 & 1.272 & $<0.30>0.20$ \\
\hline 7. & $\begin{array}{l}\mathrm{A} \rightarrow \mathrm{C} \\
\text { Low dust exposure with cough }\end{array}$ & 0.071 & 0.095 & 7 & 1.979 & $<0.10>0.05$ \\
\hline 8. & $\begin{array}{l}\mathrm{A} \rightarrow \mathrm{C} \\
\text { High dust exposure with cough }\end{array}$ & 0.178 & 0.407 & 6 & 1.072 & $<0.40>0.30$ \\
\hline
\end{tabular}

$\mathrm{A}$ and $\mathrm{B} \rightarrow$ First day (Monday) before shift and First day after shift.

$\mathrm{C}$ and $\mathrm{D} \rightarrow$ Last day (Saturday) before shift and Last day after shift.

$\overline{\mathrm{X}}=$ Mean value,$\quad \mathrm{SD}=$ Standard deviation, $\quad \mathrm{n}=$ Number of workers, $t=' t$ ' value, $\quad P=$ Level of significance.

Table 6. Changes in $\mathrm{FEV}_{1}$ in relation to smoking

\begin{tabular}{|c|c|c|c|c|c|c|}
\hline $\begin{array}{l}\text { Sr. } \\
\text { No. }\end{array}$ & Decrease in $\mathrm{FEV}_{1}$ & $\overline{\mathrm{X}}$ & $\mathrm{SD}$ & $\mathrm{n}$ & $t$ & $P$ \\
\hline 1. & $\begin{array}{l}\mathrm{A} \rightarrow \mathrm{B} \\
\text { Non-smokers }\end{array}$ & 0.105 & 0.180 & 15 & 2.251 & $<0.05>0.02$ \\
\hline 2. & $\begin{array}{l}\mathrm{A} \rightarrow \mathrm{B} \\
\text { Smokers }\end{array}$ & -0.027 & 0.364 & 17 & 0.313 & $<0.80>0.70$ \\
\hline 3. & $\begin{array}{l}\mathrm{C} \rightarrow \mathrm{D} \\
\text { Non-smokers }\end{array}$ & 0.043 & 0.099 & 15 & 1.691 & $<0.20>0.10$ \\
\hline 4. & $\begin{array}{l}\mathrm{C} \rightarrow \mathrm{D} \\
\text { Smokers }\end{array}$ & -0.019 & 0.242 & 17 & 0.320 & $<0.80>0.70$ \\
\hline 5. & $\begin{array}{l}\mathrm{A} \rightarrow \mathrm{D} \\
\text { Non-smokers }\end{array}$ & 0.206 & 0.226 & 15 & 3.530 & $<0.01>0.001$ \\
\hline 6. & $\begin{array}{l}\mathrm{A} \rightarrow \mathrm{D} \\
\text { Smokers }\end{array}$ & 0.127 & 0.400 & 17 & 1.308 & $<0.30>0.20$ \\
\hline 7. & $\begin{array}{l}\mathrm{A} \rightarrow \mathrm{C} \\
\text { Non-smokers }\end{array}$ & 0.163 & 0.219 & 15 & 2.885 & $<0.02>0.01$ \\
\hline 8. & $\begin{array}{l}\mathrm{A} \rightarrow \mathrm{C} \\
\text { Smokers }\end{array}$ & 0.139 & 0.467 & 17 & 1.231 & $<0.30>0.20$ \\
\hline
\end{tabular}

A and B $\rightarrow$ First day (Monday) before shift and First day after shift.

$\mathrm{C}$ and $\mathrm{D} \rightarrow$ Last day (Saturday) before shift and Last day after shift.

$\overline{\mathrm{X}}=$ Mean value,$\quad \mathrm{SD}=$ Standard deviation, $\quad \mathrm{n}=$ Number of workers,

$t=$ ' $t$ ' value, $\quad P=$ Level of significance. 


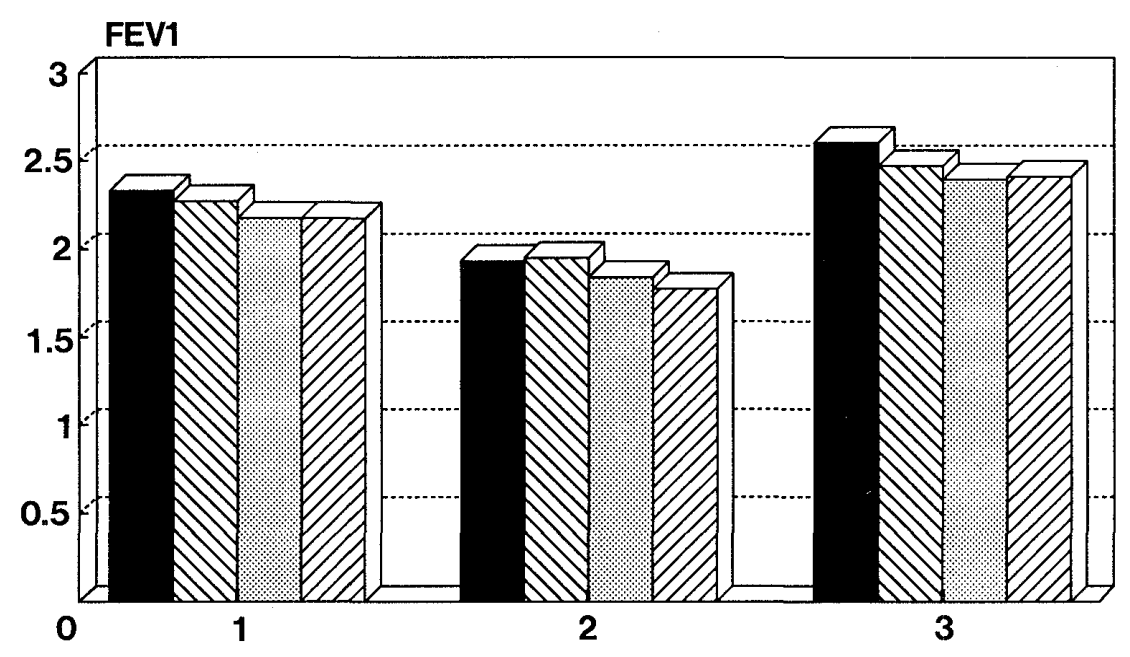

Fig. 1. Changes in $\mathrm{FEV}_{1}$ over a working week for high dust and low dust exposed groups and for the entire series.

1. Workers of the entire series.

2. High dust exposed workers.

3. Low dust exposed workers.

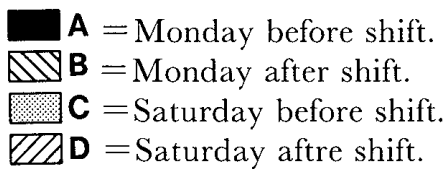

workers to the high dust exposure group. In the high dust exposure group [10], the mean difference of values are too low and sometimes the after shift values are a little higher but still statistically non-significant. In the low dust exposure group, except for the changes of last day before shift and after shift, all the other three differences were statistically significant $(108 \mathrm{ml}, 164 \mathrm{ml}$ and 183 $\mathrm{ml}$ respectively). Seven persons from the low dust exposure group and six persons from the high dust exposure group were observed to have a productive cough (Table 5). Among the differences, only the Monday before shift and Monday after shift $(145 \mathrm{ml})$ were found statistically significant. An inconclusive finding was observed in the high dust exposure group. Further study in terms of possible relevant change of the mean $\mathrm{FEV}_{1}$ over a working week for the the exposed workers is therefore necessary. The results are presented diagramatically in Fig. 1. Influence of smoking on $\mathrm{FEV}_{1}$ was presented in Table 6. In non-smokers the difference between Monday before shift and after shift are statistically significant, but in smokers these comparisons between the shift values were found to be statistically nonsignificant.

Table 7 shows that in the low dust exposure group, there are 9 nonsmokers and 11 smokers. The workers having a smoking history were found to have significant differences in these comparisons 7.6 and 7.8 , but in nonsmokers the only statistically significant difference was noticed in the comparison 7.5, with the others being non-significant. Changes of the mean 
Table 7. Changes in $\mathrm{FEV}_{1}$ in relation to low dust exposure and smoking habit

\begin{tabular}{|c|c|c|c|c|c|c|}
\hline $\begin{array}{l}\text { Sr. } \\
\text { No. }\end{array}$ & Decrease in $\mathrm{FEV}_{1}$ & $\overline{\mathrm{X}}$ & $\mathrm{SD}$ & $\mathrm{n}$ & $t$ & $P$ \\
\hline 1. & $\begin{array}{l}\mathrm{A} \rightarrow \mathrm{B} \\
\text { Low dust exposed non-smokers }\end{array}$ & 0.043 & 0.172 & 9 & 0.754 & $<0.50>0.40$ \\
\hline 2. & $\begin{array}{l}\mathrm{A} \rightarrow \mathrm{B} \\
\text { Low dust exposed smokers }\end{array}$ & 0.163 & 0.249 & 11 & 2.162 & $<0.10>0.05$ \\
\hline 3. & $\begin{array}{l}\mathrm{C} \rightarrow \mathrm{D} \\
\text { Low dust exposed non-smokers }\end{array}$ & .0 .014 & 0.087 & 9 & 0.498 & $<0.70>0.60$ \\
\hline 4. & $\begin{array}{l}\mathrm{C} \rightarrow \mathrm{D} \\
\text { Low dust exposed smokers }\end{array}$ & -0.057 & 0.249 & 11 & 0.763 & $<0.50>0.40$ \\
\hline 5. & $\begin{array}{l}A \rightarrow D \\
\text { Low dust exposed non-smokers }\end{array}$ & 0.100 & 0.156 & 9 & 1.917 & $<0.10>0.05$ \\
\hline 6. & $\begin{array}{l}\mathrm{A} \rightarrow \mathrm{D} \\
\text { Low dust exposed smokers }\end{array}$ & 0.215 & 0.211 & 11 & 3.390 & $<0.01>0.001$ \\
\hline 7. & $\begin{array}{l}\mathrm{A} \rightarrow \mathrm{C} \\
\text { Low dust exposed non-smokers }\end{array}$ & 0.085 & 0.150 & 9 & 1.707 & $<0.20>0.10$ \\
\hline 8. & $\begin{array}{l}\mathrm{A} \rightarrow \mathrm{C} \\
\text { Low dust exposed smokers }\end{array}$ & 0.263 & 0.296 & 11 & 2.939 & $<0.02>0.01$ \\
\hline
\end{tabular}

A and B $\rightarrow$ First day (Monday) before shift and First day after shift.

$\mathrm{C}$ and $\mathrm{D} \rightarrow$ Last day (Saturday) before shift and Last day after shift.

$\overline{\mathrm{X}}=$ Mean value,$\quad \mathrm{SD}=$ Standard deviation, $\quad \mathrm{n}=$ Number of workers, $t=$ ' $t$ ' value, $\quad P=$ Level of significance.

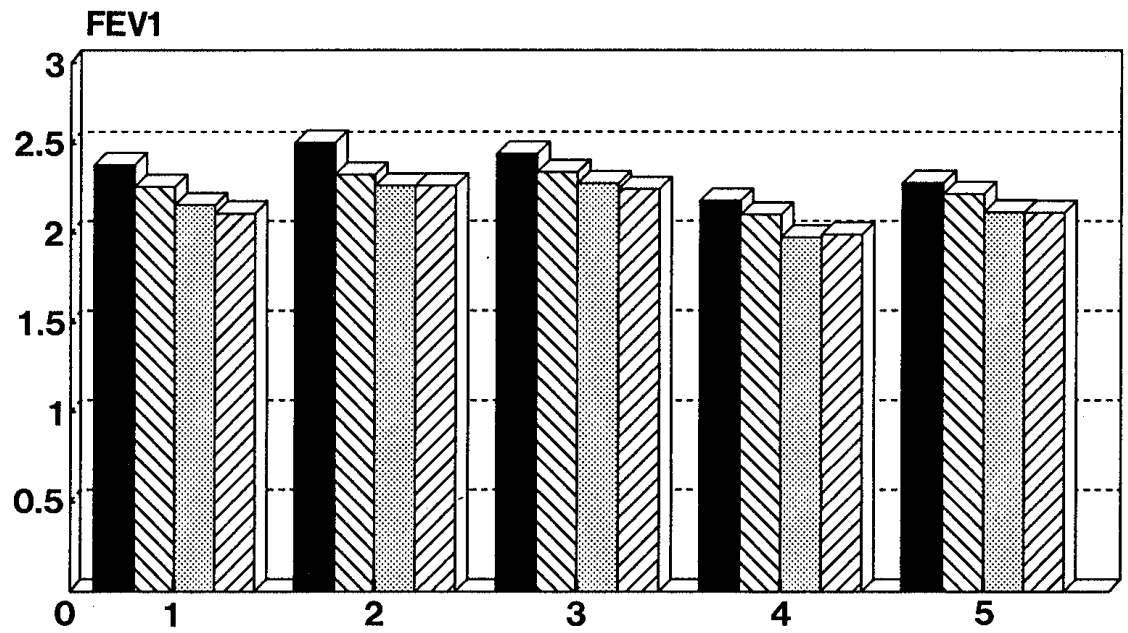

Fig. 2. Changes in mean $\mathrm{FEV}_{1}$ over a working week for workers with or without a cough and with or without a smoking habit, and the workers of the entire series.

1. Workers having cough.

2. Workers without cough.

3. Workers without smoking habit.

4. Workers with smoking habit.

5. Workers of the entire series.
$\mathbf{A}=$ Monday before shift.

MID = Monday after shift.

\% $\mathbf{C}=$ Saturday before shift.

$\square Z \mathbf{D}=$ Saturday after shift.

$\mathrm{FEV}_{1}$ over a working week for non-smokers, smokers, workers having a cough and without a cough and the workers of the entire series are presented in Fig. 2. Results of the high dust exposure group with a smoking history is presented in Table 8. It was found that Monday before shift and after shift 
Table 8. Changes in $\mathrm{FEV}_{1}$ in relation to high dust exposure and smoking habit

\begin{tabular}{|c|c|c|c|c|c|c|}
\hline $\begin{array}{l}\text { Sr. } \\
\text { No. }\end{array}$ & Decrease in $\mathrm{FEV}_{1}$ & $\overline{\mathrm{X}}$ & SD & $\mathrm{n}$ & $t$ & $P$ \\
\hline 1. & $\begin{array}{l}\mathrm{A} \rightarrow \mathrm{B} \\
\text { High dust exposed non-smokers }\end{array}$ & 0.196 & 0.162 & 6 & 2.974 & $<0.05>0.02$ \\
\hline 2. & $\begin{array}{l}\mathrm{A} \rightarrow \mathrm{B} \\
\text { High dust exposed smokers }\end{array}$ & -0.220 & 0.432 & 6 & 1.247 & $<0.30>0.20$ \\
\hline 3. & $\begin{array}{l}\mathrm{C} \rightarrow \mathrm{D} \\
\text { High dust exposed non-smokers }\end{array}$ & 0.086 & 0.108 & 6 & 1.962 & $<0.20>0.10$ \\
\hline 4. & $\begin{array}{l}\mathrm{C} \rightarrow \mathrm{D} \\
\text { High dust exposed smokers }\end{array}$ & 0.052 & 0.234 & 6 & 2.449 & $<0.10>0.05$ \\
\hline 5. & $\begin{array}{l}\mathrm{A} \rightarrow \mathrm{D} \\
\text { High dust exposed non-smokers }\end{array}$ & 0.367 & 0.231 & 6 & 3.879 & $<0.02>0.01$ \\
\hline 6. & $\begin{array}{l}\mathrm{A} \rightarrow \mathrm{D} \\
\text { High dust exposed smokers }\end{array}$ & -0.035 & 0.612 & 6 & 0.139 & $<0.90>0.80$ \\
\hline 7. & $\begin{array}{l}\mathrm{A} \rightarrow \mathrm{C} \\
\text { High dust exposed non-smokers }\end{array}$ & 0.280 & 0.267 & 6 & 2.570 & $<0.05>0.02$ \\
\hline 8. & $\begin{array}{l}\mathrm{A} \rightarrow \mathrm{C} \\
\text { High dust exposed smokers }\end{array}$ & -0.086 & 0.653 & 6 & 0.324 & $<0.80>0.70$ \\
\hline
\end{tabular}

$\mathrm{A}$ and $\mathrm{B} \rightarrow$ First day (Monday) before shift and First day after shift.

$\mathrm{C}$ and $\mathrm{D} \rightarrow$ Last day (Saturday) before shift and Last day after shift.

$\overline{\mathrm{X}}=$ Mean value,$\quad \mathrm{SD}=$ Standard deviation, $\quad \mathrm{n}=$ Number of workers, $t={ }^{\prime} t$ ' value, $\quad P=$ Level of significance.

Table 9. Changes in $\mathrm{FEV}_{1}$ in relation to cough and smoking

\begin{tabular}{|c|c|c|c|c|c|c|}
\hline $\begin{array}{l}\text { Sr. } \\
\text { No. }\end{array}$ & Decrease in $\mathrm{FEV}_{1}$ & $\overline{\mathrm{X}}$ & SD & $\mathrm{n}$ & $t$ & $P$ \\
\hline 1. & $\begin{array}{l}\mathrm{A} \rightarrow \mathrm{B} \\
\text { Cough symptoms as a whole }\end{array}$ & 0.012 & 0.230 & 13 & 0.180 & $<0.90>0.80$ \\
\hline 2. & $\begin{array}{l}\mathrm{A} \rightarrow \mathrm{B} \\
\text { Cough without smoking }\end{array}$ & 0.140 & 0.197 & 4 & 1.418 & $<0.30>0.20$ \\
\hline 3. & $\begin{array}{l}\mathrm{A} \rightarrow \mathrm{B} \\
\text { Cough with smoking }\end{array}$ & -0.046 & 0.230 & 9 & 0.594 & $<0.60>0.50$ \\
\hline 4. & $\begin{array}{l}\mathrm{C} \rightarrow \mathrm{D} \\
\text { Cough symptoms as a whole }\end{array}$ & 0.040 & 0.170 & 13 & 0.849 & $<0.50>0.40$ \\
\hline 5. & $\begin{array}{l}\mathrm{C} \rightarrow \mathrm{D} \\
\text { Cough without smoking }\end{array}$ & 0.072 & 0.123 & 4 & 1.178 & $<0.40>0.30$ \\
\hline 6. & $\begin{array}{l}\mathrm{C} \rightarrow \mathrm{D} \\
\text { Cough with smoking }\end{array}$ & 0.025 & 0.192 & 9 & 0.399 & $<0.70>0.60$ \\
\hline 7. & $\begin{array}{l}\mathrm{A} \rightarrow \mathrm{D} \\
\text { Cough symptoms as a whole }\end{array}$ & 0.181 & 0.286 & 13 & 2.275 & $<0.05>0.02$ \\
\hline 8. & $\begin{array}{l}\mathrm{A} \rightarrow \mathrm{D} \\
\text { Cough without smoking }\end{array}$ & 0.202 & 0.238 & 4 & 1.697 & $<0.20>0.10$ \\
\hline 9. & $\begin{array}{l}\mathrm{A} \rightarrow \mathrm{D} \\
\text { Cough with smoking }\end{array}$ & 0.171 & 0.318 & 9 & 1.614 & $<0.20>0.10$ \\
\hline 10 & $\begin{array}{l}\mathrm{A} \rightarrow \mathrm{C} \\
\text { Cough symptoms as a whole }\end{array}$ & 0.121 & 0.277 & 13 & 1.571 & $<0.20>0.10$ \\
\hline 11. & $\begin{array}{l}\mathrm{A} \rightarrow \mathrm{C} \\
\text { Cough without smoking }\end{array}$ & 0.101 & 0.320 & 9 & 0.948 & $<0.40>0.30$ \\
\hline 12. & $\begin{array}{l}\mathrm{A} \rightarrow \mathrm{C} \\
\text { Cough with smoking }\end{array}$ & 0.130 & 0.210 & 4 & 1.241 & $<0.40>0.30$ \\
\hline
\end{tabular}

$A$ and $B \rightarrow$ First day (Monday) before shift and First day after shift.

$\mathrm{C}$ and $\mathrm{D} \rightarrow$ Last day (Saturday) before shift and Last day after shift.

$\overline{\mathrm{X}}=$ Mean value,

$\mathrm{SD}=$ Standard deviation,

$\mathrm{n}=$ Number of workers,

$t={ }^{\prime} t$ ' value,

$P=$ Level of significance. 
(8.1), Monday before shift and Saturday after shift (8.5) and Monday before shift and Saturday before shift values (8.7) of the high dust exposed non-smoker group had significant differences but in the smoker group no significant difference was observed. Interrelationship of cough with smoking history is tabulated in Table 9 .

\section{Discussion}

The results of the present study indicated a mean decrease in $\mathrm{FEV}_{1}$ in workers exposed predominantly to jute dust at the first day and last day of work after a weekend break. Detailed analysis revealed that this decrease was virtually confined to those who were observed to have a cough along with a smoking habit and exposed to dust. Among smokers, the before shift and after shift values are less than those of non-smokers. Mean values of all PET parameters measured are also lower in smokers in comparison to nonsmokers. It was also found that the ventilatory capacity was reduced due to smoking and dust exposure.

Two other earlier studies on ventilatory capacity in jute workers during exposure have been reported. Mair et al. [5] found no significant changes in ventilatory capacity in eight jute workers during Monday and Thursday. Gilson et al. [13] also found no significant changes in 20 men during an unusually dusty Monday shift. Five of these men working in some of the dustier areas had symptoms of bronchitis, but they also showed no significant or consistent change in ventilatory capacity. Our findings are not in serious conflict with these results. It is, of course, possible that there are differences in the severity of dust exposure. Gandevia and Milne, [7] studied the cotton and jute industries and established that cotton dust is the more potent and consistent in its effect on the ventilatory capacity during a shift. Our results of jute workers may perhaps be viewed as a further illustration of a general principle which may be extended to include the possible interaction between environmental pollution and smoking in the development of ventilatory impairment. It also follows from this reasoning that it may be easier in an environment relatively free of pollution to isolate the effects of occupational exposure and to illustrate the relevance of such factors as smoking and productive cough to the ventilatory response.

Valic and Zuskin [8] reported that jute dust may cause a persistent cough and persistent phlegm. Their results suggest that jute dust is capable of causing a decrease in ventilatory capacity over a working day. The present investigation was in accordance with their results. Regarding the change of pulmonary function values according to exposure, it appears that as years of 
exposure to jute dust increased the values reduced [14]. However, in the present study, the difference between the mean values for those who have minimal and those who have maximal exposure are statistically nonsignificant. Valic and Zuskin [8] also suggested that jute dust, although nonbyssinogenic, is capable of causing a decrease in ventilatory capacity over a working day. Subjects having a persistent cough and persistent phlegm have significantly higher reductions in $\mathrm{FEV}_{1}$ than those without such symptoms. Gandevia and Milne [7] reported the significant decrement of $\mathrm{FEV}_{1}$ on the first working day. The same authors also reported that in six non-smokers employed for less than a month, there was a significant mean $\mathrm{FEV}_{1}$ decrement of $140 \mathrm{ml}$ over the first working shift after the weekend. In the present study the mean decrement of ventilatory capacity was found on the first day of work. Taking into account a very similar dust concentration, cotton dust appears to be more active in causing respiratory disease and acute lung function changes than jute dust [8]. Yet the effect of jute dust cannot be disregarded. Ghawabi [4] stated that there is no doubt that exposure to a high concentration of jute dust represents an occupational health hazard. It caused an acute reduction in ventilatory capacity during the shift that was considerably more pronounced in those who had chronic bronchitis. Jute dust exposure did not induce byssinosis but did lead to the development of chronic respiratory impairment [4]. The processing of vegetable fibres like St. Helena, Hemp, Manilla and Jute carries no specific hazards of byssinosis, although their dust may cause a chronic cough and sputum and a fall in $\mathrm{FEV}_{1}$ during the work shift [15]. The particles are large enough to be trapped in the nose and upper respiratory system. Only the small dust particles reach the lungs and contribute to the causes of certain non-specific lung diseases. Although the etiologic agent of lung disease in vegetable dusts is unclear, mineral impurities, special chemical components of fibres and micro-organisms are commonly believed to be the main etiologic factors [16]. In softening of jute fibres, some oil and water is used and the jute is stored for 48 hours in a warm house at 25 ${ }^{\circ} \mathrm{C}$. Bacteria and endotoxins may be produced by this time [16]. The endotoxin and the jute dust caused the obstructive type of lung function changes which were measured by $\mathrm{FEV}_{1}$. Ghawabi [4] reported a higher prevalence of chronic bronchitis and other obstructive types of respiratory symptoms in exposed workers compared to control. Rylander and Morey [17] stated that $\mathrm{FEV}_{1}$ is the most commonly used parameter to measure the airflow obstruction caused by dust and endotoxins. The same author has reported that the airborne lipoproteins in jute dust is $0.02-0.05 \mu \mathrm{g} / \mathrm{m}^{3}$ which is less than that of cotton dust, but may cause this type of changes. Zenlin and Zhou [18] stated that jute workers had significantly higher annual decrements of FVC, 
$\mathrm{FEV}_{1}$, FEF $25-75 \%$ than the control workers. Long term exposure to jute dust produces chronic loss of lung function.

Exposure to cotton dust and also to jute dust caused a significant reduction of $\mathrm{FEV}_{1}, \mathrm{FVC}$ and PEFR on the first working shift of the week. The measurements of $\mathrm{FEV}_{1}$ and PEFR in jute workers with and without respiratory symptoms have shown a greater mean reduction over the shift in FEV $\mathrm{F}_{1}$ Our results indicated a mean decrease in $\mathrm{FEV}_{\mathrm{l}}$ in workers exposed predominantly to jute dust during the the first day and last day of the working week. The significant deterioration of lung function in the low dust exposure group was found when compared between the before shift and after shift values that might be due to fibre fraction of dust which was found more in the low dust zone in comparison to the high dust zone [10]. The liberation of endotoxins from the fibres and the deposition of jute dust caused the obstruction of airflow, which reduced the $\mathrm{FEV}_{1}$. Further detailed study is needed to better understand the etiology of jute dust induced lung diseases. It might be the inference of the view that some exposure to dust was necessary to produce the observed falls in $\mathrm{FEV}_{1}$. It is now generally accepted that PET decrement will occur in an average working population due to occupational hazards, smoking habit and cough symptoms.

\section{Acknowledgments}

The authors are grateful to the Director, National Institute of Occupational Health, Ahmedabad, for his kind permission and encouragement in conducting this study. We are also grateful to the subjects for their active co-operation in the smooth conduction of our investigation. Our thanks are also due to Mr. S. Thakur for statistical analysis and Mr. A. K. Dey for secretarial assistance.

\section{References}

1. Arlidge J T (1892): The Hygiene, Disease and Mortality of Occupations Percival, London. (Cited from Valic, F. \& Zuskin, E. 1971. A comparative study of respiratory function in female non smoking cotton and jute workers. Brit J Industr Med 28: 364-368)

2. Schilling R S F (1956): Byssinosis in cotton and other textile workers. Lancet-2 261-265, 319-325. (Cited from Valic F \& Zuskin E 1971 Brit J Industr Med 28: 364-368)

3. Suddhu C M S, Nath K, Mehorta P K (1968): Byssinosis amongst cotton and jute workers in Kanpur. Ind J Med Res 54: 980-993

4. Ghawabi S H EL (1978): Respiratory function and symptoms in workers exposed simultaneously to jute and hemp. J Industr Med 35: 16-20

5. Mair A, Smith, D H, Wilson W A et al (1960): Dust disease in Dundee textile workers. Brit J Industr Med 17: 272-278 
6. Popa V, Gavrilescu N, Preda N et al (1969): An investigation of allergy in byssinosis sensitization to cotton, hemp, flax and jute antigens. Brit J Industr Med 26: 101-108

7. Gandevia B \& Milne J (1965): Ventilatory capacity on exposure to jute dust and the relevance of productive cough and smoking to the response. Brit $\mathrm{J}$ (1965): Ventilatory capacity on exposure to jute dust and the relevance of productive cough and smoking to the response. Brit J Intern Med 22: 187-195

8. Valic F \& Zuskin E (1971): A comparative study of respiratory function in female nonsmoking cotton and jute workers. Brit J Industr Med 28: 364-368

9. Morgan K C \& Seaton A (1984): Occupational Lung Diseases. 2nd ed. W B Saunders, Philadephia p 541

10. Mukherjee A K, Bhattacharya S K, Ahmad S et al (1991): An environmental survey in jute processing industry. Ind J Industr Med 37 (3): 123-128

11. Threshold limit values and biological exposure indices (1986): American Conference of Governmental Industrial Hygienists 1986-87

12. Du-Bois D \& Du-Bous E F (1916): Clinical Calorimetry: A formulae to estimate the approximate body surface area if height and weight be known. Arch Intern Med 17: 863-871

13. Gilson J C, Stott H, Hopwood B E C et al (1962): Byssinosis. The acute effect on ventilatory capacity of dusts in cotton ginneries, cotton, sisal and jute mills. Brit $\mathrm{J}$ Industr Med 19: 9-18

14. Chattopadhyay B P, Alam Jane Sk \& Gangopadhyay P K (1989): A study of dynamic lung functions in jute mill workers. Ind J Industr Med 35: 157-165

15. Mckerrow C B, Gilson J C, Schilling R S F et al (1965): Respiratory function and symptoms in rope workers. Brit J Industr Med 22: 204

16. Zhou G, Lin Z, Ho G et al (1989): Respiratory symptoms and lung function in jute processing workers-A primary investigation. Arch Environ Health 44: 370-374

17. Rylander R \& Morey P (1982): Airborne endotoxin in industries processing vegetable fibres. Am Industr Hyg Assoc J 43: 811-812

18. Zenlin L \& Zhou C (1992): A longitudinal study of lung function in jute processing workers. Arch Environ Health 47: 218-222 
インド麻粉塵の呼吸機能に及ぼす影響と湿咳および喫煙の関連性

B. P. チヤトパジャイ, S. K. ジェンアラム, P. K. ガンゴパジャイ, H. N. セイエド

東部地域産業保健センター, カルカッタ, インド

要旨：インド麻工場での男性作業者 32 人について呼吸機能を調べた。職業歴，病歷，喫 煙歴などの他，呼吸器の症状に関する詳しい調査を行った後に肺機能のテストを行 った．作業者の中には喀痰を伴う咳をする者もみられた。週の初日と比べると週の 最終日には FEV 1 の平均値の低下がみられたので，作業の種類，作業場の粉塵の多 少，あるいは喫煙習慣などとの関連性を調べた. FEV 1 の低下が粉塵の多い作業場 で働く人，哭煙量の多い人，湿咳のある人に多くみられたが，これらの因子との関 連性に関しては統計学的には有意ではなかった．粉塵濃度の低い作業場で働く人で 湿咳㧍よび猰煙歴をもつ作業者で仕事の後で $\mathrm{FEV}_{1}$ の顕著な減少がみられた。これ らの因子の肺機能に及ぼす影響を考察した。

J UOEH（産業医大誌）17 (2 ): 91-104 (1995) 